\title{
Onset Detection to Study Muscle Activity in Reaching and Grasping Movements in Rats
}

\author{
Yolanda Castillo-Escario, María Rodríguez-Cañón, Guillermo García-Alías, Raimon Jané, \\ Senior Member, IEEE
}

\begin{abstract}
EMG signals reflect the neuromuscular activation patterns related to the execution of a certain movement or task. In this work, we focus on reaching and grasping (R\&G) movements in rats. Our objective is to develop an automatic algorithm to detect the onsets and offsets of muscle activity and use it to study muscle latencies in R\&G maneuvers.

We had a dataset of intramuscular EMG signals containing 51 R\&G attempts from 2 different animals. Simultaneous video recordings were used for segmentation and comparison. We developed an automatic onset/offset detector based on the ratio of local maxima of Teager-Kaiser Energy (TKE). Then, we applied it to compute muscle latencies and other features related to the muscle activation pattern during $R \& G$ cycles. The automatic onsets that we found were consistent with visual inspection and video labels. Despite the variability between attempts and animals, the two rats shared a sequential pattern of muscle activations. Statistical tests confirmed the differences between the latencies of the studied muscles during $R \& G$ tasks.

This work provides an automatic tool to detect EMG onsets and offsets and conducts a preliminary characterization of muscle activation during $R \& G$ movements in rats. This kind of approaches and data processing algorithms can facilitate the studies on upper limb motor control and motor impairment after spinal cord injury or stroke.
\end{abstract}

\section{INTRODUCTION}

Electromyographic signals (EMG) measure the electrical activity generated during muscle contraction. Studying EMG recordings during the execution of voluntary movements helps to understand the neuromuscular activation patterns related to this task. This can elucidate the movement control structure directed by the central nervous system (CNS), and how it can be affected by neuromuscular disorders.

In this work, we study the reaching and grasping (R\&G) movement in rats. This upper limb ability allows reaching external objects and interacting with them. Therefore, it

*Research supported by a fellowship from "la Caixa" Foundation (ID 100010434, fellowship code LCF/BQ/ES18/11670019), Government of Catalonia (CERCA Program), Secretaria d'Universitats i Recerca del Departament d'Empresa i Coneixement de la Generalitat de Catalunya (Consolidated research group GRC 2017 SGR 01770), Neuron-ERA-NET program and Instituto Carlos III (AC16/00050) to GGA.

Y. Castillo-Escario and R. Jané are with the Institute for Bioengineering of Catalonia (IBEC), the Barcelona Institute of Science and Technology (BIST), Centro de Investigación Biomédica en Red de Bioingeniería, Biomateriales y Nanomedicina (CIBER-BBN) and ESAII Department, Universitat Politècnica de Catalunya - BarcelonaTech (UPC), Barcelona, Spain (e-mail: \{ycastillo, rjane\}@ibecbarcelona.eu).

M. Rodríguez-Cañon is with IBEC, Institute of Neurosciences, Universitat Autònoma de Barcelona (UAB) and Centro de Investigación Biomédica en Red de Enfermedades Neurodegenrativas (CIBER-NED), Barcelona, Spain

G. García-Alías is with UAB, CIBER-NED and the Guttmann Institute, Institut Universitari de Neurorehabilitació, Badalona, Spain constitutes a fundamental motor skill for the survival of many animal species. On the other hand, $R \& G$ movement is highly stereotyped and conserved in mammals, what makes it an interesting translational model for therapy research [1]. One of its applications is the study of upper limb motor impairment and recovery after a cerebrovascular accident, spinal cord injury (SCI) or other neuromuscular disorders. However, there is little research on EMG patterns during upper limb movements. Just a few studies have recently started focusing on this field, proposing some tools to analyze and categorize the EMG activity during R\&G [2], or pointing to alterations in the EMG activity of forelimb muscles after SCI in rats [3]. This work is aimed to be the first proof of concept to define the R\&G cycle in rats using EMG.

The study of EMG is complex due to its stochastic nature and susceptibility to interferences [4], which results in a low signal-to-noise ratio (SNR). A major challenge when working with EMG signals is onset and offset detection, i.e. to determine the start and end instants of muscular activity. This topic has great interest in many research and clinical fields. It has long been studied but remains an open question. In fact, many different algorithms have been published for this purpose, but there is no unique solution. It rather depends on the specific application.

The most simplistic way to label onsets and offsets is through visual inspection. However, this is prone to human errors, time-consuming and unfeasible for large data analysis. Moreover, apart from the intrinsic subjectivity of this method, it is not an easy task to manually identify muscle activity regions, as EMG is a weak and noisy signal. Common automatic approaches use EMG energy information (e.g. RMS - root mean square) and apply a single or double threshold, estimated from the signal. Other approaches include the use of sample entropy or statistical criterion determination, but these require a priori knowledge of the signal [4].

The Teager-Kaiser Energy (TKE) [5-6] is a non-linear operator which may improve the accuracy of onset detection. It has been tested in both surface [7] and intramuscular EMG [4]. Its advantage is that it is proportional to the instantaneous amplitude and frequency of the signal, thus emphasizing motor unit action potentials (MUAPs) and improving SNR [7]. After applying the TKE operator to the signal, the onset is usually computed as the instant when the TKE exceeds a certain value, as in conventional thresholding methods. This value is calculated from the mean and standard deviation of background noise in TKE domain.

Here we present a method for onset detection based on the TKE. However, instead of using a threshold, we propose 
an alternative algorithm, based on the ratios of consecutive local maxima in TKE domain, which highlight amplitude variations. The second part of this work is aimed at using the automatically detected onsets to characterize muscle latencies during reaching and grasping movements in rats.

\section{MATERIALS AND METHODS}

\section{A. Experiments and Data Acquisition}

All experimental procedures were performed in compliance with the University's Chancellor's Animal Research Committee and complied with the guidelines of the National Institutes of Health. We worked with a database of intramuscular EMG signals from two Long Evans rats. Electrodes were implanted in five muscles of the rats preferred paw to reach and grasp: middle deltoid, biceps brachii, pronator teres, extensor digitorum communis (EDC) and flexor digitorum superficialis (FDS).

During the experiments, the animals were individually placed inside a cage with a small window. Food pellets were presented, and the rats had to extend their paw through the window and take the pellet, thus completing a R\&G cycle.

EMG signals were acquired at a sampling frequency of 10 $\mathrm{kHz}$ and gain 1000, using a multi-channel analog amplifier. A video was recorded simultaneously at 30 frames/second. It was used to manually segment the signals, by selecting the frames where the R\&G cycles started (when the animal paw was lifted) and ended (when the pellet was released in the mouth). The fast movements complicated this detection, so a tolerance of two frames was used to ensure that the actual start and end of the $\mathrm{R} \& \mathrm{G}$ cycle were inside these windows [2]. A 4-frames segment ( $130 \mathrm{~ms})$ before the start of each cycle was taken as a measure of background noise.

\section{B. Signal Processing}

Initially, we had 60 attempts of $R \& G$ (30 from each rat). Poor-quality signals and attempts where the EMG of some muscle had not been correctly recorded were discarded after visual inspection. A total number of 51 attempts remained (25 from rat 1 and 26 from rat 2). The duration of these cycles was $0.6 \pm 0.2 \mathrm{~s}$ (mean \pm standard deviation).

Signals were imported to Matlab (r2018a, Mathworks Inc.), where they were processed and analyzed through custom-made algorithms. Signals were resampled to $3 \mathrm{kHz}$, using an anti-aliasing low-pass filter. Then, they were bandpass filtered $(20-1000 \mathrm{~Hz})$ with an $8^{\text {th }}$ order Butterworth filter, in both forward and reverse directions. A comb-notch filter was applied to remove power-line noise and harmonics.

EMG signals were rectified and TKE was computed and used as a signal envelope, as explained below.

\section{Onset/Offset Detection}

The TKE operator was applied to the signals of each $\mathrm{R} \& \mathrm{G}$ attempt. For a given signal, $x(n)$, the TKE, $\Psi$, can be calculated as:

$$
\Psi(\mathrm{n})=\mathrm{x}^{2}(\mathrm{n})-\mathrm{x}(\mathrm{n}+1) \mathrm{x}(\mathrm{n}-1)
$$

The obtained $\Psi(n)$ was low-pass filtered using a $2^{\text {nd }}$ order Butterworth filter with a cut-off frequency of $15 \mathrm{~Hz}$. We used this filtered signal to work in the TKE domain.
We identified TKE local maxima and computed the amplitude ratios of consecutive peaks, to highlight amplitude variations. The highest ratios should correspond to EMG onsets, due to the sharp transitions between baseline and muscle activity. Therefore, we sorted the ratios in descending order, keeping a maximum of 5 ratios, but discarding those $<2$. Onsets were defined as the instant corresponding to the local minima between the two peaks of the remaining ratios.

For each onset, the corresponding offset was the next point where the signal went below the amplitude level of the onset. If an offset was not found before the next onset, this next onset was removed. If there was no offset before the end of the signal, it was set to the last time point. Finally, to avoid the effect of short impulsive artifacts, activities shorter than a certain length (we used $50 \mathrm{~ms}$ ) were removed.

Several activity regions can be defined if more than one onset is found by the detector. Regions which are very close to each other could be combined, but we preferred to keep this information of muscle activation / inactivation. In subsequent analysis we only use the first onset and last offset, so it does not matter for this application.

\section{Feature Extraction and Statistical Analysis}

After detecting the onsets of the 5 muscles for each attempt, the first onset was selected and considered as the beginning of the R\&G cycle. The end of the cycle was set at the last offset of the 5 muscles, although it always coincides with the video label. These automatic estimations of the start and end points of the R\&G cycle were compared with the actual events, indicated by the 2 -frame windows in the video.

A time normalization was applied to bring all the attempts to an equivalent domain. From that moment, the time scale was no longer in seconds, but in percentage of the $R \& G$ cycle. Thus, it went from $0 \%$ (the time when the R\&G cycle started, i.e. the first muscle onset) to $100 \%$ (the time when the R\&G finished, i.e. the last muscle offset).

Muscle latencies were calculated as the distance from the beginning of the R\&G cycle to the onset of each muscle EMG (in \% cycle). On the other hand, we computed the cumulative energy curve of each muscle as the cumulative sum of its TKE envelope at each time point. The last value corresponds to the total energy of this muscle in the $R \& G$ cycle, which depends on the amplitude and duration of muscle activity. We calculated the so-called "center of gravity" (CoG) of the cumulative energy curve, as the time point accumulating $50 \%$ of the energy (i.e. the time when the energy curve reaches or exceeds $50 \%$ of the total energy).

Nonparametric statistical tests (Wilcoxon-Mann-Whitney two-sample rank-sum test) were used to compare latencies and CoGs between different muscles and between the two animals.

\section{RESUltS \& DiscUSSION}

\section{A. Onset/Offset Detection}

We implemented our algorithm to detect the onsets and offsets of all the muscles at each attempt. An example can be seen in Fig. 1, showing the EMG of the five muscles during a $\mathrm{R} \& \mathrm{G}$ cycle, together with the calculated onsets and offsets. 

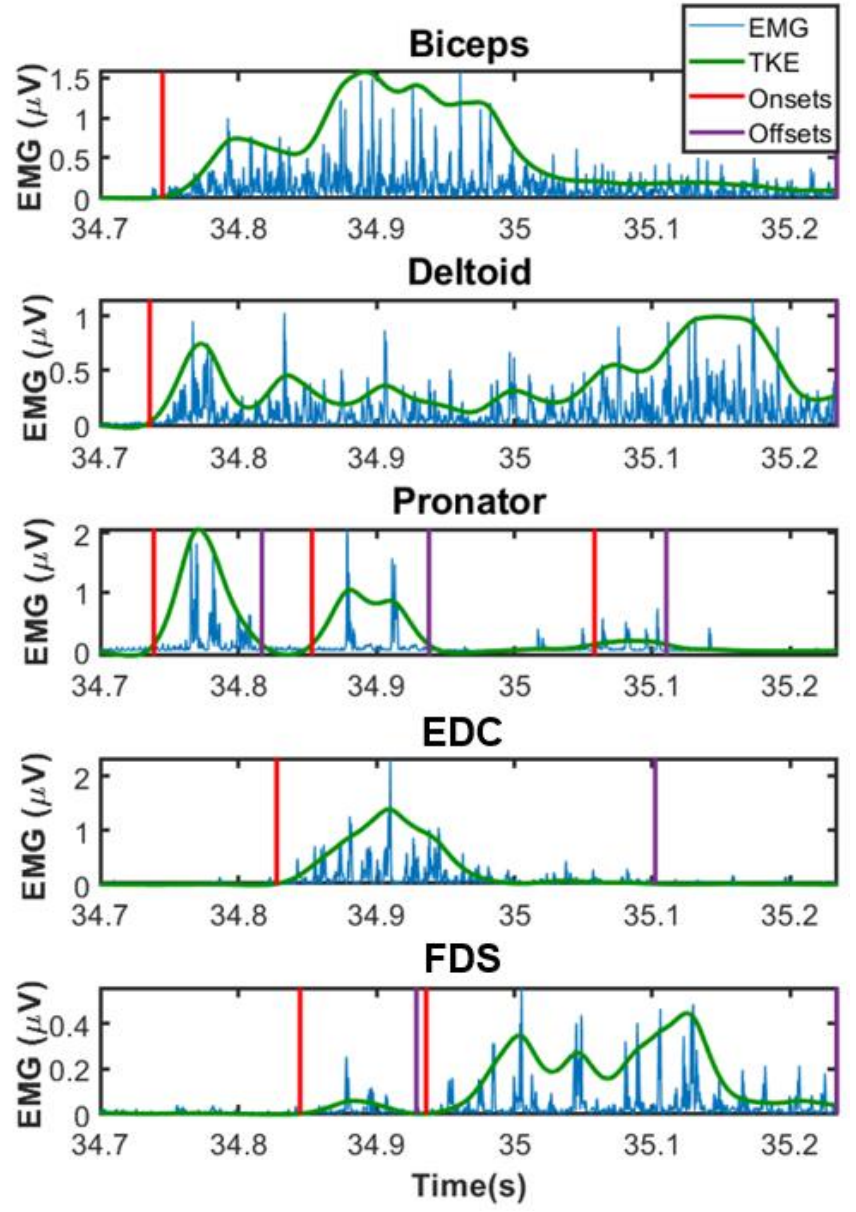

Figure 1. Example of a reaching and grasping attempt (rat 1), showing the rectified EMG of the five muscles (blue), the TKE envelopes (green) and automatic labels of onsets (red) and offsets (purple).

At each attempt, the onset of the first muscle to be activated was taken as the start of the R\&G cycle. In all cases (51 attempts), this point was inside the window from the video indicating when the rat lifted its paw. Therefore, the error of the automatic method is lower than the window length, set a priori to $1 / 15 \mathrm{~s} \approx 67 \mathrm{~ms}$, although it represents around $10 \%$ of the $R \& G$ cycle.

The same happened when comparing the last automatic offset to the window in the video indicating the end of the movement. Nevertheless, it is important to notice that, in all the attempts, the last offset found by the automatic algorithm was at the end of the segment, since some of the muscles remain active even after the $R \& G$ movement is over. This happens because the animal's upper limb is not at rest after the pellet retrieval. For instance, in Fig. 1 we can see that the last offsets of biceps, deltoid and FDS are at the end of the segment, since these muscles remain activated. Thus, further characterization of the EMG patterns would be required to determine whether it is possible to automatically establish the end of the R\&G cycle using other features and methods.

While we can define the start and end times of the R\&G using the video, EMG analysis allows us to go further and detect the specific activation times of each muscle. We had no standard measure to test the performance of our algorithm in this regard, but results were manually reviewed, and they largely agreed with visual inspection. On the other hand, our method avoids certain problems of classical approaches, such as the need for a threshold or adaption to each muscle [8].

Small inaccuracies could be attributed to the smoothing effects introduced by filters, or to residual activities (either artifacts or MUAPs). In these cases, onset detection is complex, even by visual inspection. An example can be seen in Fig. 1 with the spike-like EMG of pronator. This muscle commonly shows shorter and less dense activity regions, so the smoothed TKE envelope does not fit the muscle activity pattern as properly as in other muscles.

Our sample is limited to two rats, so the performance of the algorithm should be accurately tested with more examples and different conditions, including noisier or pathological signals. Nonetheless, the set of tools we developed to detect the onsets of muscular activity can be a first step to enable an automatic definition of $R \& G$ cycles in large EMG databases.

\section{B. Muscle Latencies}

Muscle latencies show when each muscle is activated during a certain movement or task. Therefore, they indicate the sequence of muscles activation and reflect co-activations (i.e. simultaneous activation of several muscles). This information about muscular temporal activation can help to understand the movement and the functional role of each anatomical component.

We used the automatically detected onsets of each muscle to study their latencies from the beginning of $R \& G$ maneuvers. A boxplot of the five muscles latencies can be seen in Fig. 2, separately for the two animals. Circles inside the boxes indicate mean values. It shows that biceps, deltoid and pronator are always the first muscles to be activated, followed by EDC, and finally FDS. Specifically, pronator is the muscle with less variability and a lower mean value, starting the R\&G movement in most attempts. Although there are clear differences between the two animals, this sequence of activation is conserved.

Statistically significant differences were found between all the muscles latencies in both rats ( $\mathrm{p}$-values $<0.01)$. When comparing the two animals, statistical tests indicate differences in the latencies of biceps, deltoid, pronator and FDS, but not in the EDC (Fig. 2).

We also computed the times when each muscle accumulated $50 \%$ of its total energy (CoGs) (Fig. 3). This shows similar information than muscle latencies, but instead of measuring just the first time point when the muscle is activated, it provides information about its energy distribution, i.e. its evolution along the R\&G cycle.

Fig. 3 shows that CoGs do not exactly follow the same organization than muscle latencies. This suggests that, although muscles are firstly activated in a certain sequence, their activity distribution is more variable. Nevertheless, in both animals, pronator tends to reach its $\mathrm{CoG}$ before EDC, and EDC before FDS. It is an evidence of this sequential pattern (pronator-EDC-FDS), which could already be guessed from Fig. 1 and emerges again in Fig. 2-3. A visual examination of all the attempts agrees with this fact, as already described in [2]. While biceps and deltoid have a variable and spread activity pattern along the entire R\&G 
cycle, the other muscles behavior is much more delimited and repeatable: pronator is the first to be activated, followed by EDC and finally FDS. This suggests that the differences between these two animals lie in the recruitment of proximal muscles (biceps and deltoid, whose activities are compensated), while they share a common temporal recruitment pattern of the three distal muscles (pronator, EDC and FDS).

Statistics indicate significant differences between the two rats in biceps and EDC muscles (both had a lower CoG in rat 1). Regarding intra-subject comparisons, significant differences were found between each pair of muscles, except for those pointed by yellow and orange brackets in Fig. 3 . Differences between muscles were expected, since each one has its own function as part of the movement, so they are activated or reach the $50 \%$ of their total energy in different times of the $R \& G$ cycle. These times are very repetitive, and they are characteristic of each muscle. Of course, there is a natural variability across the results, both between animals and between attempts of the same animal. These attempts included both successes and failures in grasping the pellet (it sometimes fell from the animal's hand). Although the movement sequence was visually the same, this is clearly a source of variability. However, variability can also be attributed to the different strategies that the animals can adopt to perform the same movement, according to their previous experience and the specific situation.

\section{CONCLUSION}

This work provides automatic tools to study onset and offset of muscular activity in EMG signals, muscle latencies and muscle activity patterns. On the one hand, we proposed a novel method to detect muscular activity onsets and offsets; on the other, we computed some features to start characterizing muscular activity patterns during R\&G movements in rats. These solutions are scalable to other movements and applications.

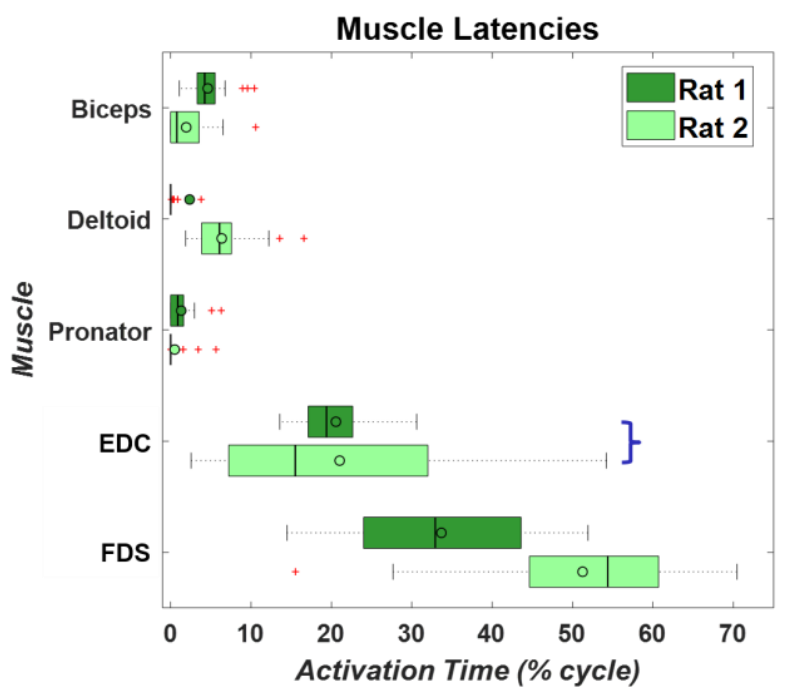

Figure 2. Boxplot of muscle latencies during R\&G for the 5 muscles and the 2 animals. Red crosses are outliers. Brackets indicate muscles with NO significant differences between the 2 rats ( $\mathrm{p}$-values $>0.01)$.
Sample size should be increased to optimize data processing algorithms and study whether it is possible to generalize the muscle patterns found for these two animals or, on the contrary, inter-subject variability plays a key role and the movement is highly subject-specific.

Intramuscular EMG signals analysis has allowed us to study neuromuscular circuits in vivo in freely moving animals. Furthermore, these techniques could potentially be transferred to surface EMG in human subjects. Future work may help to understand the motor control structure related to $\mathrm{R} \& \mathrm{G}$ movements in healthy animals, but also in injured rats with hemiplegia produced by spinal cord injury, stroke or other neuromuscular disorders. Such information will be of great clinical value to understand neural plasticity and motor recovery and develop new neurorehabilitation strategies.

\section{REFERENCES}

[1] A. Klein, L.A. Sacrey, I.Q. Whishaw and S.B. Dunnett, "The use of rodent skilled reaching as a translational model for investigating brain damage and disease", Neurosci Biobehav Rev, vol. 36, no. 3, pp. 1030-1042, Mar 2012

[2] M. Rodríguez-Cañón, I. Delgado, R. Jané and G. García-Alías, "Temporal categorization of upper limb muscle's EMG activity during reaching and grasping", ICNR 2018, Biosysrob vol. 21, L. Masia et al. Ed. Springer, 2019, pp.876-679

[3] M. Alam et al., "Electrical neuromodulation of the cervical spinal cord facilitates forelimb skilled function recovery in spinal cord injured rats", Exp Neurol, vol. 291, pp. 141-150, May 2017

[4] D. Yang, H. Zhang, Y. Gu and H. Liu, "Accurate EMG onset detection in pathological, weak and noisy myoelectric signals", Biomed Signal Proces, vol. 33, pp. 306-315, Mar 2017

[5] H. M. Teager, "Evidence for nonlinear sound reduction mechanisms in the vocal tract", Kluwer Acad Publ, pp. 241-261, 1990

[6] J. F. Kaiser, "On a simple algorithm to calculate the energy of a signal", IEEE Int Conf Acoustic Speech Signal Process, Albuquerque, NM, USA, pp. 381-384, Apr 1990

[7] X. Li, P. Zhou and A. S. Aruin, "Teager-Kaiser energy operation of surface EMG improves muscle activity onset detection", Ann Biomed Eng, vol. 35, no. 9, pp. 1532-1538, Sep 2007

[8] M. Rodríguez, R. Jané and G. García, "Temporal recruitment of forelimb proximal and distal muscles during reaching and grasping", CASEIB Proceedings, pp. 89-92, 2017

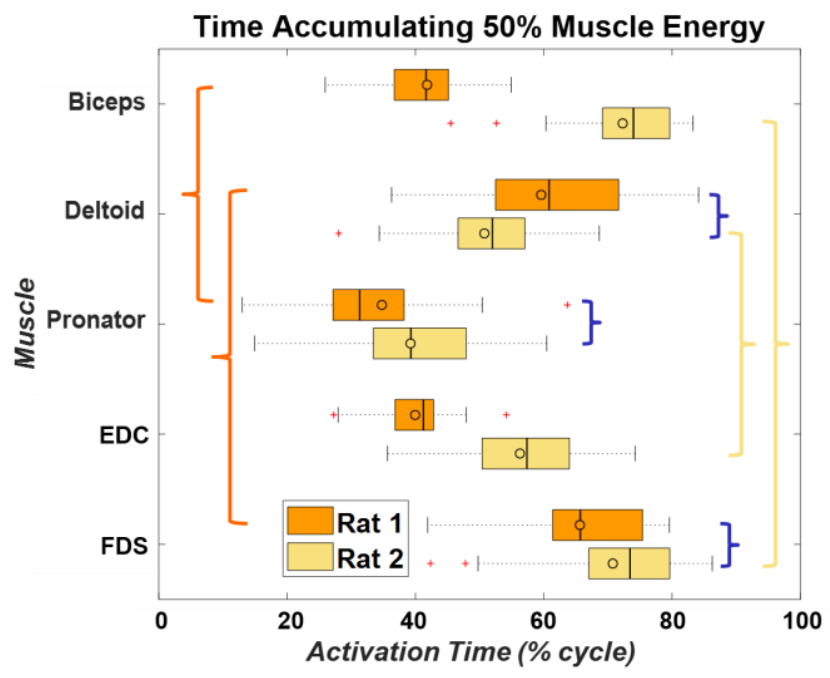

Figure 3. Boxplot of times accumulating the $50 \%$ of the total muscular energy during R\&G, for the 5 muscles and the 2 animals. Blue brackets indicate NO significant differences between the 2 rats, while orange and yellow brackets indicate NO significant differences between these muscles in rat 1 or 2 , respectively ( $\mathrm{p}$-values $>0.01$ ). 